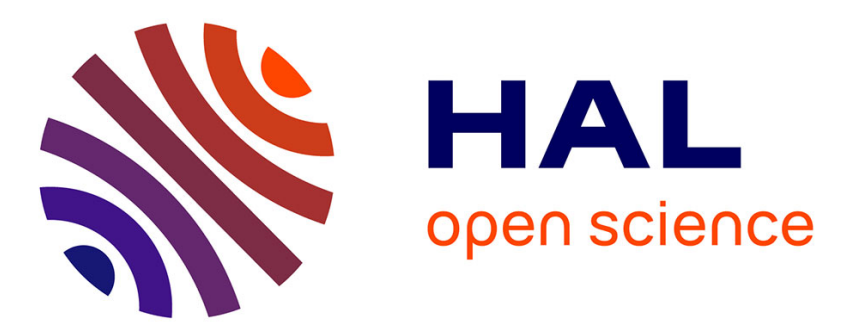

\title{
Spreading fronts in sedimentation of dilute suspension of spheres
}

Daniel Chehata Gomez, Laurence Bergougnoux, Elisabeth Guazzelli, E. John Hinch

\section{> To cite this version:}

Daniel Chehata Gomez, Laurence Bergougnoux, Elisabeth Guazzelli, E. John Hinch. Spreading fronts in sedimentation of dilute suspension of spheres. Physics of Fluids, 2008, 20, pp.023302. $10.1063 / 1.2883960$. hal-01066988

\section{HAL Id: hal-01066988 \\ https://hal.science/hal-01066988}

Submitted on 22 Sep 2014

HAL is a multi-disciplinary open access archive for the deposit and dissemination of scientific research documents, whether they are published or not. The documents may come from teaching and research institutions in France or abroad, or from public or private research centers.
L'archive ouverte pluridisciplinaire HAL, est destinée au dépôt et à la diffusion de documents scientifiques de niveau recherche, publiés ou non, émanant des établissements d'enseignement et de recherche français ou étrangers, des laboratoires publics ou privés. 


\title{
Spreading fronts in sedimentation of dilute suspension of spheres
}

\author{
Daniel Chehata Gómez, ${ }^{1}$ Laurence Bergougnoux, ${ }^{1}$ Élisabeth Guazzelli, ${ }^{1}$ and John Hinch ${ }^{2}$ \\ ${ }^{1}$ IUSTI CNRS UMR 6595, Polytech'Marseille, Technopôle de Château-Gombert, \\ 13453 Marseille Cedex 13, France \\ ${ }^{2}$ DAMTP, University of Cambridge, Wilberforce Road, Cambridge CB3 OWA, United Kingdom
}

(Received 18 May 2007; accepted 24 January 2008; published online 29 February 2008)

\begin{abstract}
The thickness of the diffuse front between a sedimenting dilute suspension and the clear fluid above grows linearly in time due to polydispersity in the size of the particles and due to a hydrodynamic effect in which randomly heavy clusters fall out of the front leaving it depleted. Experiments and simplified point-particle numerical simulations agree that these two effects are not simply linearly additive. () 2008 American Institute of Physics. [DOI: 10.1063/1.2883960]
\end{abstract}

\section{INTRODUCTION}

The settling of particles in a viscous fluid is a phenomenon extensively studied because it is found in a variety of natural or biological situations, such as the flow of sediment in rivers or the flow of red blood cells, and in industrial processes where it is commonly used to separate particles of different sizes or densities. The mean sedimentation velocity of a suspension can be predicted theoretically at low particle volume fraction ${ }^{1}$ and by using sophisticated computer simulations at larger volume fractions, but the individual movement of particles due to long-range hydrodynamic interactions gives rise to velocity fluctuations that are still not completely understood. ${ }^{2-13}$ Lately, the study of the spreading of the sedimentation front has regained interest ${ }^{12,13}$ as it has been advocated that a small vertical stratification due to the broadening of this front can change the characteristics of the velocity fluctuations. ${ }^{10-12}$

One can find a few experimental investigations of the spreading of this front in the literature showing the importance of different effects, such as hydrodynamic dispersion, polydispersity of the particles, and hindered settling. Davis and Hassen ${ }^{14}$ were the first to demonstrate that the observed spreading of the front could not be explained by the sole effect of polydispersity but should also be attributed to hydrodynamic dispersion. For more concentrated suspensions, the self-sharpening effect strongly reduces the sedimentation interface. Lee et al. ${ }^{15}$ established that dispersion and selfsharpening must be taken into account simultaneously to describe the front spreading and infer the variation of the dispersion coefficient as a function of particle volume fraction. Martin et al. ${ }^{16}$ showed that when hydrodynamic dispersion and self-sharpening effects are both included, the sedimentation front profile has a stationary shape propagating with a constant velocity.

To summarize the experimental results, the front spreading seems to be controlled by hydrodynamic dispersion at early times causing the front to grow diffusively with the square root of time. Later, the front grows linearly in time due to the effect of polydispersity. Hindered settling effects can then lead to an equilibrium thickness. In recent experiments at low volume fraction, Bergougnoux et al. ${ }^{13}$ found that the sedimentation front grows linearly in time. They were able to explain $75 \%$ of this spreading by polydispersity but the other $25 \%$ remained not completely elucidated. It was found difficult to deconvolve the simultaneous actions of polydispersity and other small effects such as hydrodynamic dispersion and flux enhancement due to density fluctuations. This last effect is new and has not been studied before. The initial mixing of the suspension creates density fluctuations, also called "blobs," on all length-scales (from container size down to the mean interparticle spacing), which give rise to convection currents, also called "swirls," on all length-scales. The heavy blobs fall faster and leave behind the interface region depleted of particles, thus increasing the spreading of the front.

Computer simulations can easily suppress the experimental complication of polydispersity as well as the hindered settling effects by simplifying the hydrodynamic interactions between the particles. ${ }^{12,13}$ However, it is still unclear how the front will grow with time. Two models have been proposed. The first model ${ }^{13}$ considers heavy blobs of the scale of the mean interparticle spacing falling faster and emptying the interface, which produces a linear growth of the thickness of the front with time. The second model ${ }^{12}$ considers a nonlinear hydrodynamic dispersion due to swirls, the size of which is limited by stratification, and leads to a thickness of the front growing in time as $t^{5 / 7}$. The simulation data were unable to distinguish between these two models.

In this paper, we examine both by experiments and numerical simulations the effect of container size, polydispersity, and volume fraction on the spreading of the sedimentation front. The aim of the study is to discriminate between the different effects of polydispersity, hydrodynamic dispersion, and flux enhancement due to density fluctuations.

\section{EXPERIMENTAL TECHNIQUES}

Five different batches of glass spheres, supplied by Cataphote Inc. and Potters Industries, were used to perform the experiments. The particle size distributions were measured using a CCD digital camera attached to a microscope and the public domain Java image processing program IMAGEJ. ${ }^{17}$ From measurements of the projected bead surfaces, the particle-radius distributions were observed to be approximately Gaussian for all the different batches and 
TABLE I. Particle characteristics.

\begin{tabular}{lccccc}
\hline \hline Batch & $\begin{array}{c}\langle a\rangle \\
(\mathrm{cm})\end{array}$ & $\begin{array}{c}\sigma_{a} /\langle a\rangle \\
(\%)\end{array}$ & $\begin{array}{c}\rho_{p} \\
\left(\mathrm{~g} / \mathrm{cm}^{3}\right)\end{array}$ & $\begin{array}{c}V_{\mathrm{S}} \\
(\mathrm{cm} / \mathrm{s})\end{array}$ & $\begin{array}{c}\mathrm{Re}_{p} \\
\times 10^{-5}\end{array}$ \\
\hline $\mathrm{A}(\bigcirc)$ & 0.0202 & 2.5 & $2.50 \pm 0.04$ & $0.0136 \pm 0.0009$ & 2.7 \\
$\mathrm{~B}(\square)$ & 0.0152 & 2.5 & $4.11 \pm 0.07$ & $0.0158 \pm 0.0010$ & 2.3 \\
$\mathrm{C}(\triangle)$ & 0.0148 & 5.6 & $4.11 \pm 0.07$ & $0.0150 \pm 0.0018$ & 2.1 \\
$\mathrm{D}(\diamond)$ & 0.0187 & 11.3 & $2.50 \pm 0.04$ & $0.0117 \pm 0.0027$ & 2.1 \\
$\mathrm{E}(\nabla)$ & 0.0229 & 19.7 & $2.50 \pm 0.04$ & $0.0175 \pm 0.0070$ & 3.9 \\
\hline \hline
\end{tabular}

therefore well represented by a mean radius $\langle a\rangle$ and a standard deviation $\sigma_{a}$ (see Table I). The particle density $\rho_{p}$ was determined by measuring the volume variation when a weighted amount of particles were introduced into a known volume of distilled water in a graduated vessel and is also indicated in Table I. The fluid used was silicone oil 47V1000, supplied by Rhodia, which had a viscosity $\mu=10.0 \pm 0.3 \mathrm{P}$ and a density $\rho_{f}=0.965 \pm 0.007 \mathrm{~g} \mathrm{~cm}^{-3}$ at the air-conditioned room temperature of $25 \pm 1{ }^{\circ} \mathrm{C}$. The particle characteristics, the Stokes velocity of an isolated sphere $V_{\mathrm{S}}$, and the sphere Reynolds number $\operatorname{Re}_{p}=V_{S}\langle a\rangle \rho_{f} / \mu$ are listed in Table I. The Péclet number was always very large for these combinations of particles and fluid.

Experiments were performed in glass-wall vessels of different cross sections $\left(20 \times 20 \mathrm{~cm}^{2}, 10 \times 10 \mathrm{~cm}^{2}\right.$, and $4 \times 10 \mathrm{~cm}^{2}$ ) but filled with the same fluid height of $40 \mathrm{~cm}$. A CCD digital camera (Basler A102f) with a wide-angle lens was positioned on a rail in front of the sedimentation vessel and imaged the whole cell. The cell was backlit by two neon tubes. Tracing papers were placed on the back face of the vessel to homogenize the illumination. Lateral walls were covered with sheets of black paper in order to avoid lateral sources of light.

The above setup was used to measure the light attenuation produced by the sedimenting suspension, following the same approach as Bergougnoux et al. ${ }^{13}$ First, the calibration law giving the light attenuation as a function of the mean particle volume fraction $\phi_{0}$ was determined. The light attenuation averaged on each pixel horizontal line, i.e., $I_{0}(\zeta)$, along the suspension height $\zeta$ was measured through pure fluid; i.e. for zero volume fraction. The same measurement was performed with a well-mixed suspension at a given volume fraction $\phi_{0}$ yielding the intensity $I\left(\zeta, \phi_{0}\right)$ for each horizontal line. The relative light attenuation averaged over the whole height of the cell defined as $\left\langle I / I_{0}\right\rangle\left(\phi_{0}\right)$ was found to be a monotonically decreasing function of $\phi_{0}$. Using this calibration curve, the time variation of the volume fraction $\phi(t, \zeta)$ was then determined at different horizontal pixel lines located at the different heights $\zeta$. In fact, as the intensity signal is noisy because the particle images are of the order of one pixel, we performed a moving average over ten horizontal pixel lines, which smooths the concentration measurement.

Each experiment consisted of first mixing the suspension and then recording the whole image of the sedimenting suspension every $40 \mathrm{~s}$ until the upper front reached the bottom of the cell. For achieving a homogeneous suspension, a small propeller fixed to a drill motor was used. The mixing process lasted for several minutes until a homogeneous suspension was observed through the whole height. The mixing procedure was the same for all the experiments. The starting time $(t=0)$ was defined as the cessation of mixing.

Three different types of experiments were performed to characterize the spreading of the front. First, we examine the influence of the cell size and shape using particles of batch $\mathrm{C}$ at $\phi_{0}=0.3 \%$. Secondly, we considered the effect of polydispersity using the different batches of particles while keeping the volume fraction constant at $\phi_{0}=0.3 \%$ and using the same cell of square cross section $10 \times 10 \mathrm{~cm}^{2}$. Thirdly, the effect of concentration was studied using particles of batch $B$ in the cell of square cross section $10 \times 10 \mathrm{~cm}^{2}$. The concentration was varied from $0.1 \%$ to $0.8 \%$ by steps of $0.1 \%$.

\section{EXPERIMENTAL RESULTS}

The spreading of the front was measured using the approach of Davis and Hassen ${ }^{14}$ revisited by Bergougnoux et $a l .{ }^{13}$ The curves $\phi(t, \zeta)$ obtained by the light attenuation technique explained in the preceding section II are used to determine the interface median, first, and third quartile times, $t_{1 / 2}, t_{1 / 4}$, and $t_{3 / 4}$, respectively, corresponding to the time taken by the isoconcentration planes at $\phi(t) / \phi_{0}=\frac{1}{2}, \frac{1}{4}$, and $\frac{3}{4}$, respectively, to fall a distance $\zeta$. Typical plots of the dimensionless falling distance $\zeta /\langle a\rangle$ as a function of dimensionless time $t / t_{\mathrm{S}}$ [time in units of the Stokes time $t_{\mathrm{S}}=\langle a\rangle / V_{\mathrm{S}}$ ] are given in Figs. 1(a) and 1(b). A clear linear increase with time is found for all the experiments. The slopes of these straight lines are obtained by using linear fittings and correspond to the velocities $w_{1 / 2}, w_{1 / 4}$, and $w_{3 / 4}$ of the isoconcentration planes $\phi(t) / \phi_{0}=\frac{1}{2}, \frac{1}{4}$, and $\frac{3}{4}$, respectively. We can also compute the interface thickness between $\frac{1}{4}$ and $\frac{3}{4}$ quartiles, i.e., $\delta=t_{1 / 2}\left(\zeta / t_{3 / 4}-\zeta / t_{1 / 4}\right)$, as a function of the falling distance $\zeta$. The half-widths $\delta_{1 / 2-1 / 4}=t_{1 / 2}\left(\zeta / t_{1 / 2}-\zeta / t_{1 / 4}\right)$ and $\delta_{3 / 4-1 / 2}=t_{1 / 2}\left(\zeta / t_{3 / 4}-\zeta / t_{1 / 2}\right)$ are also computed.

The experimental data can be compared to predictions of polydispersity neglecting concentration effects. We consider that the light attenuation technique probes the surfaces of the particles. From the measured particle-radius distribution, we can infer the distributions of the square of the radius $a$. The accumulated distributions of the square of the radius for all batches are shown in Fig. 2 . We can then deduce the median, first, and third quartile radii; i.e., $a_{1 / 2}, a_{1 / 4}$, and $a_{3 / 4}$, respectively. The median, first, and third quartile velocities, i.e., $w_{1 / 2}^{p}, w_{1 / 4}^{p}$, and $w_{3 / 4}^{p}$, due to polydispersity are simply given by the Stokes velocity computed for the median, first, and third quartile radii, respectively. The interface thickness due to polydispersity is simply given by $\delta^{p} / \zeta=\left(a_{3 / 4}^{2}-a_{1 / 4}^{2}\right) / a_{1 / 2}^{2}$. Additionally, the half-widths $\delta_{1 / 2-1 / 4}^{p} / \zeta=\left(a_{1 / 2}^{2}-a_{1 / 4}^{2}\right) / a_{1 / 2}^{2}$ and $\delta_{3 / 4-1 / 2}^{p} / \zeta=\left(a_{3 / 4}^{2}-a_{1 / 2}^{2}\right) / a_{1 / 2}^{2}$ can be computed.

Figure 3 shows that the interface thickness $\delta$ increases linearly with the settling distance $\zeta$ and that this increase is similar for experiments in different cell sizes with the same particles of batch $\mathrm{C}$ at $\phi_{0}=0.3 \%$. The relative quartile interface thicknesses $\delta / \zeta$ are given by the slopes of the straight lines obtained from linear fits and are $\approx 0.20$ for the experiments in the three different cells. The finite thickness of the 

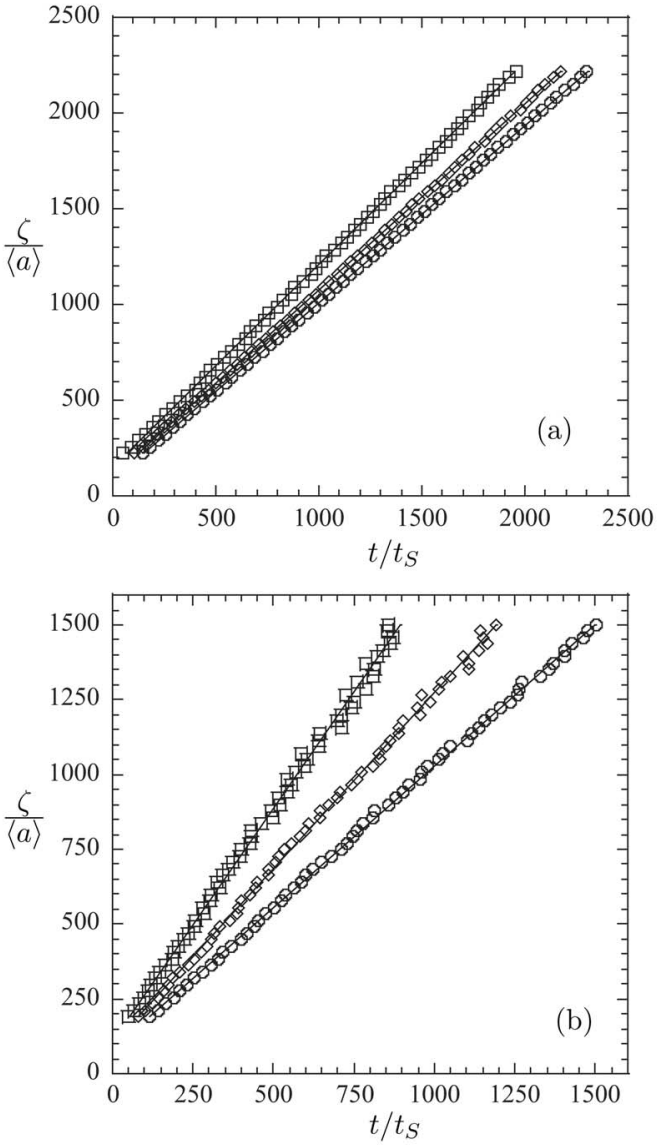

FIG. 1. Dimensionless falling distance $\zeta /\langle a\rangle$ vs dimensionless time $t / t_{S}$, for the median $(\diamond)$, first $(\bigcirc)$, and third $(\square)$ quartiles for batch B (a) and batch E (b). The different lines indicate the best linear fits. Experiments were held in a $10 \times 10 \mathrm{~cm}^{2}$ vessel. The suspension concentration was $\phi_{0}=0.3 \%$.

front at the initial moment is due to difficulties in making the suspension homogeneous to the very top while avoiding entraining air bubbles by the mixing device.

Figure 4 shows again that $\delta$ increases linearly with $\zeta$ for experiments in the same cell and at the same $\phi_{0}=0.3 \%$ but

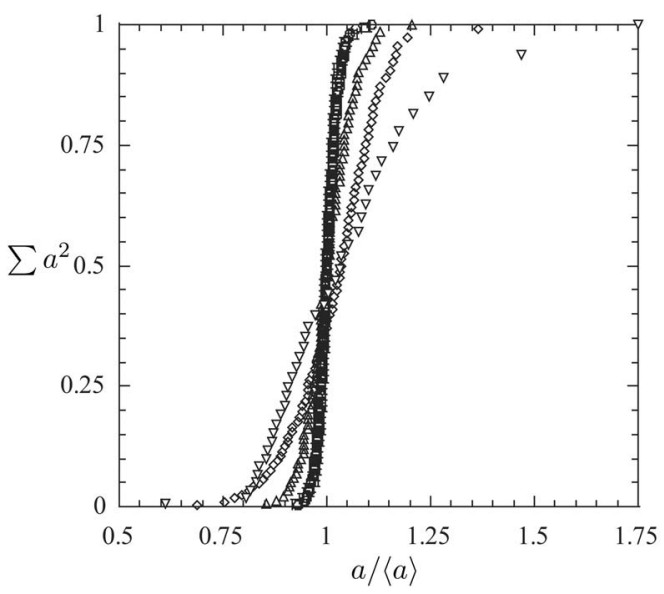

FIG. 2. Normalized cumulative sum of the square of the radius $\left(a^{2}\right)$ vs dimensionless radius $(a /\langle a\rangle)$ for the five batches of particles: A $(\bigcirc)$, B $(\square)$, $\mathrm{C}(\triangle), \mathrm{D}(\diamond)$, and $\mathrm{E}(\nabla)$.

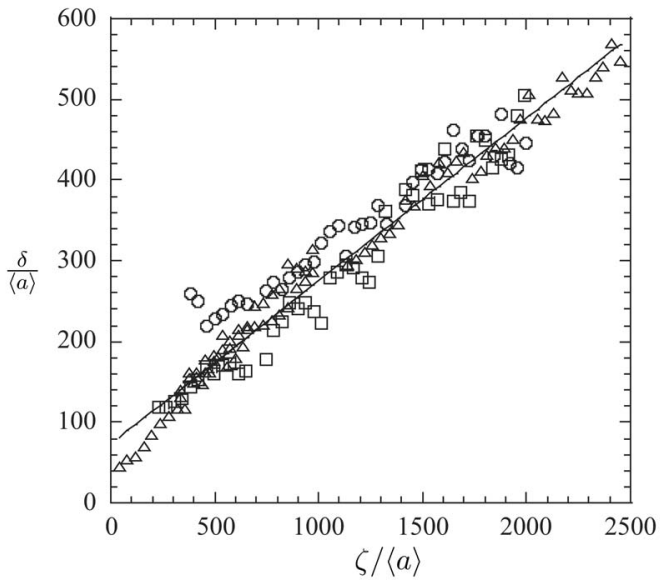

FIG. 3. Dimensionless interface thickness $\delta /\langle a\rangle$ vs dimensionless falling distance $\zeta /\langle a\rangle$, for batch $\mathrm{C}$ at $\phi_{0}=0.3 \%$. The line indicates the best linear fit. The different cross sections presented are $20 \times 20 \mathrm{~cm}^{2}(\triangle), 10 \times 10 \mathrm{~cm}^{2}$ $(\bigcirc)$, and $4 \times 10 \mathrm{~cm}^{2}(\square)$.

with batches of particles having different polydispersities. Clearly the spreading increases with increasing polydispersity. The relative quartile interface thickness $\delta / \zeta$ is compared to that predicted by polydispersity $\delta^{p} / \zeta$ in Fig. 5(a) (see also Table II). When the polydispersity is large, most of the spreading can be explained by polydispersity. Conversely, for the most monodisperse particles, the polydispersity can only explain half of the experimental spreading. A more refined examination of this effect can be obtained by comparing the experimental isoconcentration velocities with those predicted by polydispersity in Table II. In the case of the most polydisperse particles, the velocities $w_{1 / 4}$ and $w_{3 / 4}$ are equally spaced around $w_{1 / 2}$. On the contrary, for monodisperse particles, the velocity $w_{1 / 2}$ is much closer to $w_{1 / 4}$. This can also be seen in Figs. 1(a) and 1(b). In the most polydisperse case, see Fig. 1(b), the median line lies midway between the first and third quartile lines while, in the most

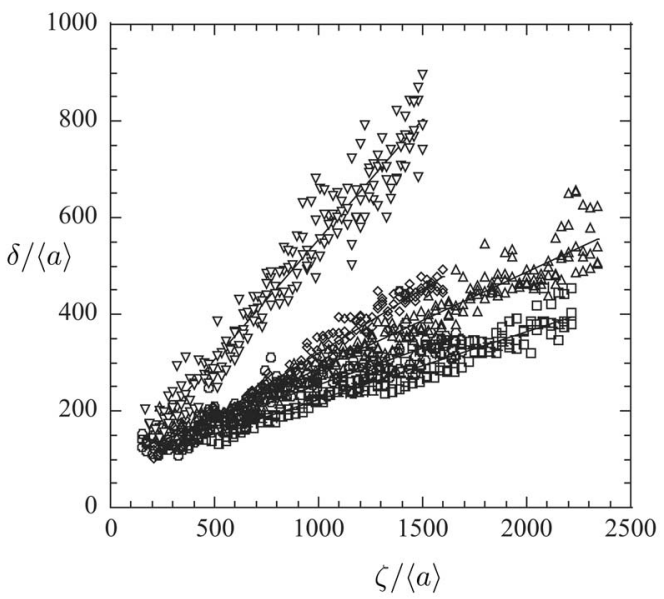

FIG. 4. Dimensionless interface thickness $\delta /\langle a\rangle$ vs dimensionless falling distance $\zeta /\langle a\rangle$. The lines indicate the best linear fits. Three different experiments were realized for each different batch of particles; A $(\bigcirc)$, B ( $\square)$, C $(\triangle), \mathrm{D}(\diamond)$, and $\mathrm{E}(\nabla)$. 

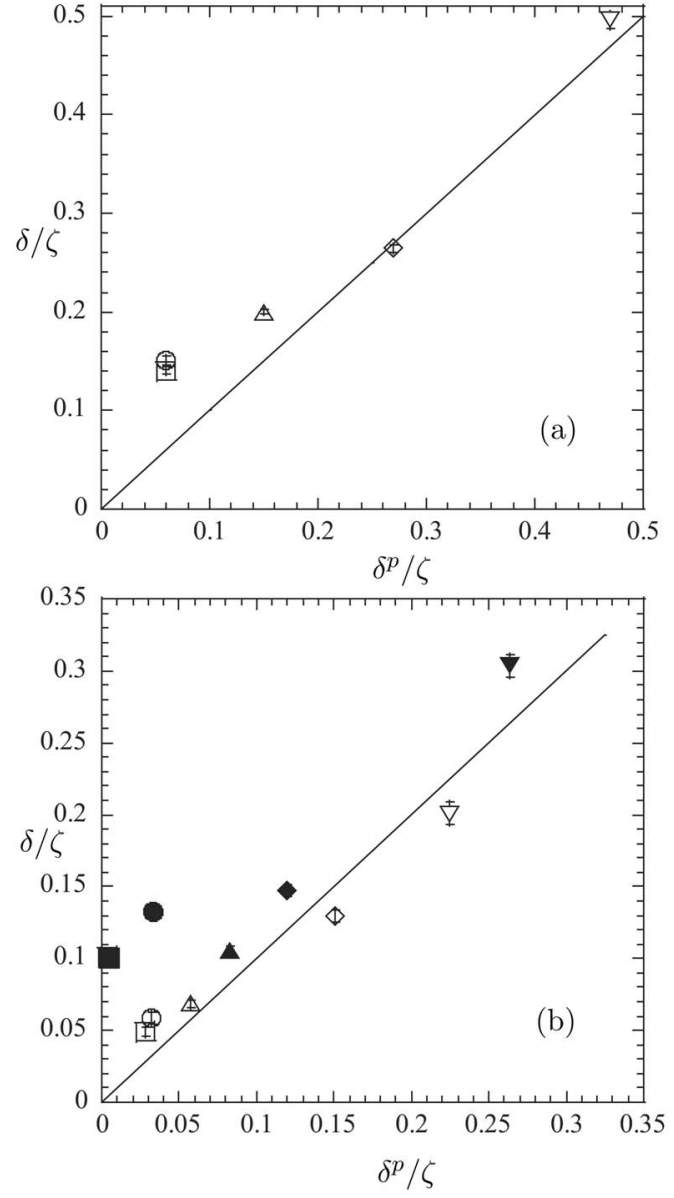

FIG. 5. (a) Dimensionless interface thickness $\delta / \zeta$ vs dimensionless predicted quartile interface $\delta^{p} / \zeta$. The symbols correspond to the batches $\mathrm{A}(\bigcirc)$, B $(\square), \mathrm{C}(\triangle), \mathrm{D}(\diamond)$, and E $(\nabla)$. (b) Dimensionless half-widths $\delta_{1 / 2-1 / 4} / \zeta$ (open symbols) and $\delta_{3 / 4-1 / 2} / \zeta$ (filled symbols) vs dimensionless predicted half-widths $\delta_{1 / 2-1 / 4}^{p} / \zeta$ and $\delta_{3 / 4-1 / 2}^{p} / \zeta$, respectively.

monodisperse case [see Fig. 1(a)], the third quartile line is further away. This can also be seen in Fig. 5(b), which compares half-widths with those predicted by polydispersity. The $\frac{1}{2}-\frac{1}{4}$ half-width seems to be explained by polydispersity effects while the $\frac{3}{4}-\frac{1}{2}$ half-width is wider and departs from the polydispersity predictions as polydispersity is decreased.

The effect of concentration on interface spreading is examined in Fig. 6 (see also Table III). The spreading increases with increasing $\phi_{0}$ for small $\phi_{0}$, reaches a maximum at

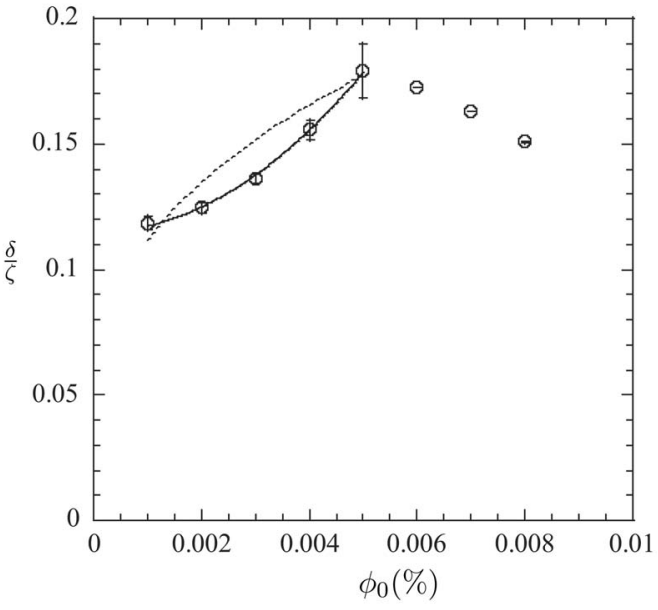

FIG. 6. Relative quartile interface thickness $\delta / \zeta$ vs the concentration $\phi_{0}$. The solid curve represents a quadratic fit to the first five concentration values. The dotted curve is explained in the conclusion.

$\approx 0.5 \%$, and then decreases. The initial increase with increasing $\phi_{0}$ can be well fitted by a $\phi^{2}$ quadratic function, as seen in the Fig. 6.

Finally, we have examined whether an universal profile of concentration in the front can be found. Figure 7 presents the concentration profile of the front as a function of $\left(\zeta-\zeta_{h}\right) / \delta$, where $\zeta_{h}$ is the height of the $\phi / \phi_{0}=\frac{1}{2}$ concentration. The data for different times collapse onto the same master curve. This is observed in Fig. 7 for the most monodisperse particles at low volume fraction, but is also seen for more polydisperse particles and at larger volume fractions, though the data are more scattered between the $\frac{3}{4}-\frac{1}{2}$ concentrations. Again, we notice that the width between the $\frac{1}{2}-\frac{1}{4}$ concentrations is smaller than that between the $\frac{3}{4}-\frac{1}{2}$ concentrations. The normalized height combination $\left(\zeta-\zeta_{h}\right) / \delta$ used in Fig. 7 is equivalent to a ratio of the velocities of different concentrations $\left[w(\phi)-w_{1 / 2}\right] /\left(w_{3 / 4}-w_{1 / 4}\right)$.

\section{COMPUTER SIMULATIONS}

Computer simulations have been made with the method of Bergougnoux et al. ${ }^{13}$ adapted to polydisperse particles. The hydrodynamic interactions are calculated treating the particles as point-particles which exert a force equal to their weight compensated for buoyancy. The induced Stokes flow

TABLE II. Dimensionless isoconcentration velocities and relative interface thicknesses deduced from the experiments and from predictions accounting solely for polydispersity for the different particle batches at $\phi_{0}=0.3 \%$. The experimental data correspond to averages over three experimental runs.

\begin{tabular}{|c|c|c|c|c|c|c|c|c|}
\hline \multirow[b]{2}{*}{ Batch } & \multicolumn{4}{|c|}{ Polydispersity predictions } & \multicolumn{4}{|c|}{ Experiments } \\
\hline & $w_{1 / 4}^{p} / V_{\mathrm{S}}$ & $w_{1 / 2}^{p} / V_{\mathrm{S}}$ & $w_{3 / 4}^{p} / V_{\mathrm{S}}$ & $\delta^{p} / \zeta$ & $w_{1 / 4} / V_{\mathrm{S}}$ & $w_{1 / 2} / V_{\mathrm{S}}$ & $w_{3 / 4} / V_{\mathrm{S}}$ & $\delta / \zeta$ \\
\hline A & 0.971 & 1.003 & 1.036 & 0.06 & 0.836 & 0.888 & 1.006 & 0.15 \\
\hline $\mathrm{B}$ & 0.971 & 0.999 & 1.029 & 0.06 & 0.923 & 0.971 & 1.069 & 0.14 \\
\hline $\mathrm{C}$ & 0.945 & 1.003 & 1.086 & 0.15 & 1.005 & 1.079 & 1.193 & 0.2 \\
\hline $\mathrm{D}$ & 0.898 & 1.057 & 1.183 & 0.27 & 0.956 & 1.098 & 1.260 & 0.26 \\
\hline $\mathrm{E}$ & 0.824 & 1.063 & 1.343 & 0.47 & 0.950 & 1.189 & 1.550 & 0.50 \\
\hline
\end{tabular}


TABLE III. Dimensionless isoconcentration velocities and quartile interface thickness deduced from the experiments for particles of batch $\mathrm{B}$ at different concentrations. The experimental data correspond to averages over three experimental runs.

\begin{tabular}{lllll}
\hline \hline$\phi_{0}(\%)$ & $w_{1 / 4} / V_{\mathrm{S}}$ & $w_{1 / 2} / V_{\mathrm{S}}$ & $w_{3 / 4} / V_{\mathrm{S}}$ & $\delta / \zeta$ \\
\hline 0.1 & 0.934 & 0.969 & 1.061 & 0.12 \\
0.2 & 0.881 & 0.921 & 1.006 & 0.13 \\
0.3 & 0.923 & 0.971 & 1.069 & 0.14 \\
0.4 & 0.919 & 0.975 & 1.085 & 0.16 \\
0.5 & 0.871 & 0.934 & 1.048 & 0.18 \\
0.6 & 0.872 & 0.934 & 1.041 & 0.17 \\
0.7 & 0.849 & 0.908 & 1.006 & 0.16 \\
0.8 & 0.818 & 0.871 & 0.956 & 0.15 \\
\hline \hline
\end{tabular}

is represented by a finite sum of Fourier modes. Each Fourier mode satisfied boundary conditions of no flow normal through the boundaries and no tangential stress; these modes being simpler than those satisfying no-slip boundary conditions (see Bergougnoux et al. ${ }^{13}$ for detailed expressions for the modes). The spatial distribution of the point-forces exerted by the distributed particles is thus broken down into appropriate Fourier modes of force which then drive the Fourier modes of the flow. This flow is evaluated at each particle in order to move the particles.

The simulations were conducted in a box of width $L$, depth $L$, and height $4 L$; the same geometry as in the majority of the experiments. The number of Fourier modes used was $n_{F}$ in each horizontal direction and $4 n_{F}$ in the vertical direction. Thus the smallest length resolved was $L / n_{F}$. In most of the simulations, the number of particles $N$ was set equal to the total number of Fourier modes; i.e., $4 n_{F}^{3}$. This means that the flow was well resolved for length-scales from the size of the box $(L)$ down to the interparticle separation $L(4 / N)^{1 / 3}$. Smaller length-scales, down to the particle diameter, were not resolved at all.

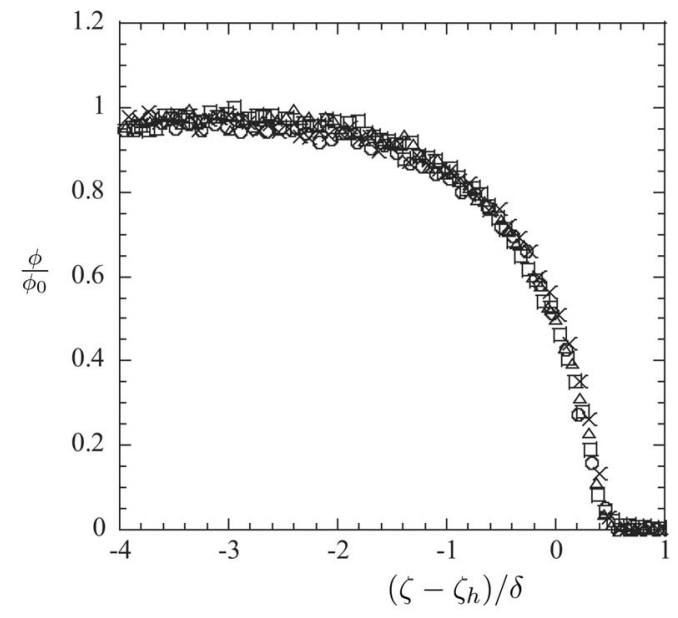

FIG. 7. Concentration profile of the sedimentation front $\phi / \phi_{0}$ vs the normalized height $\left(\zeta-\zeta_{h}\right) / \delta$. The results correspond to experiments in the $10 \times 10 \mathrm{~cm}^{2}$ cross-section vessel with particles of batch B at $\phi_{0}=0.3 \%$. The symbols correspond to the different times $t / t_{s}=418.2(\bigcirc), 669.1(\times), 961.8$ $(\triangle)$, and $1087.2(\square)$.
As a result of the limited spatial resolution, a single particle does not sediment at its correct Stokes settling velocity $F / 6 \pi \mu a$, but instead on average at $0.15 F n_{F} / \mu L$, where $F$ is the force on the particle, $\mu$ the viscosity of the fluid, and $a$ the radius of the spherical particle. In order to have the particles sediment at their correct velocity, it is therefore necessary to add a correction of $F / 6 \pi \mu a-0.15 F n_{F} / \mu L$ to the hydrodynamically induced flow from the limited number of Fourier modes. This correction introduces the parameter $a$, the "radius" of the particle. Although the "radius" exists in the expression above, it is elsewhere ignored so that the hydrodynamic interactions are calculated for point-particles, and also no effects of excluded volume are included. While the fluid does not cross the boundaries, the particles move vertically relative to the fluid due to the above sedimentation correction and thus pass through the bottom boundary. When this happens, they are immediately repositioned just above the bottom boundary.

Polydispersity in the size of the particles is introduced by assigning to each particle a random radius

$$
a=a_{0}(1+\alpha \text { rand })
$$

where the parameter $\alpha$ is the measure of the polydispersity and "rand" is a random number uniformly distributed on $\left[-\frac{1}{2}, \frac{1}{2}\right]$, and hence has zero mean and variance $\frac{1}{12}$. Associated with this radius, the force that the particle exerts is proportional to its volume, so

$$
F=F_{0}(1+\alpha \text { rand })^{3},
$$

with the same random number as in the radius. The correction to the sedimentation velocity is then

$$
V=\frac{F_{0}}{6 \pi \mu a_{0}}(1+\alpha \text { rand })^{2}-\frac{0.15 F_{0} n_{F}}{\mu L}(1+\alpha \text { rand })^{3} .
$$

The numerical simulations are nondimensionalized by scaling lengths on $L$, velocities on $F_{0} / \mu L$, and thus time on $\mu L^{2} / F_{0}$. The nondimensional sedimentation velocity then becomes

$$
V_{0}=\frac{L}{6 \pi a_{0}}
$$

While this is clearly the ratio of the size of the box to the size of the particles, we are ignoring the size of the particles except in this sedimentation velocity. In the simulations, we therefore set values of $V_{0}$ as well as the (nondimensional) polydispersity parameter $\alpha$.

The simulations use a small number of Fourier modes, with $n_{F}=5,7,10$, and 14, and corresponding number of particles $N=500,1372,4000$, and 10976 . Typical values of the nondimensional sedimentation velocity were $V_{0}=1$ to 6 . The degree of polydispersity was up to $\alpha=0.35$. For each set of these parameters, eight independent runs were made, quantities for each run being averaged over 320, 80, 20, or 10 independent realizations according to whether $n_{F}=5,7,10$, or 14. One realization involved uniformly distributing the initial positions of the particles in the box and allocating the 


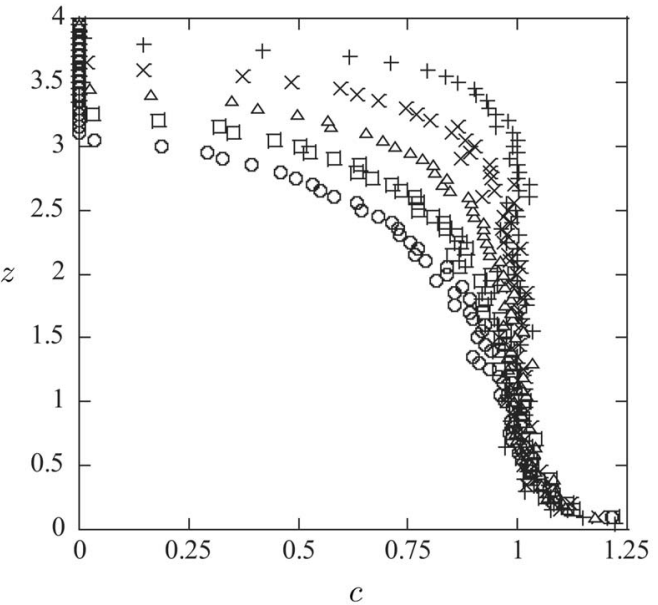

FIG. 8. Concentration profiles at $t=0.1(+), 0.2(\times), 0.3(\triangle), 0.4(\square)$, and $0.5(\bigcirc)$ for a $1 \times 1 \times 4$ box with 4000 point-particles, $10 \times 10 \times 40$ Fourier modes, sedimentation velocity $V_{0}=3.0$, and monodisperse particles $(\alpha=0)$. For this figure, an average was made over 80 realizations instead on the normal 20 for this number of particles.

random radii. From the statistical scatter among the eight runs, an estimate could be made of the likely error in any reported averaged quantity.

Initial tests were made to demonstrate that (i) the results were unchanged when the nondimensional time-step $\Delta t$ was less than $2.5 \times 10^{-3}$, (ii) the initial mean settling speed was the imposed value $V_{0}$, and (iii) when the hydrodynamic interactions were turned off, the initial rms velocity fluctuation was the imposed $(1 / \sqrt{3}) V_{0} \alpha \sqrt{1+\alpha^{2} / 60}$.

\section{NUMERICAL RESULTS}

At regular intervals of time, the vertical concentration profiles in the simulations were found by binning the vertical position of the particles in boxes of height $1 /\left(2 n_{F}\right)$. Accumulating over the different realizations, there were typically 1000 particles in each box, so the statistical fluctuations are around $3 \%$. The concentrations are plotted normalized by the initial uniform concentration. Figure 8 shows the concentration profiles at five different times during a typical simulation. At early times, there is a uniform concentration away from the top and bottom, with statistical fluctuations of a few percent. During the evolution, a sediment collects at the bottom and at the top a front descends and spreads. By $t=0.5$, the front has descended a height 1.5 corresponding to the imposed mean sedimentation velocity of 3.0. The front has spread to a thickness of around 1.0, although the leading edge of the front has just reached the sediment. Hence, the simulation was terminated at this time.

For each concentration profile, the heights were determined where the concentration was $\frac{1}{4}, \frac{1}{2}$, and $\frac{3}{4}$ of the initial uniform value. A best linear fit to $c(z)$ was made to those concentrations within $10 \%$ of the quartile value, and from this linear fit the height at which the concentration passed through the quartile was found. At very early times there were sometimes no boxes where the concentrations was within $10 \%$ of the quartile, and thus the height of the quartile

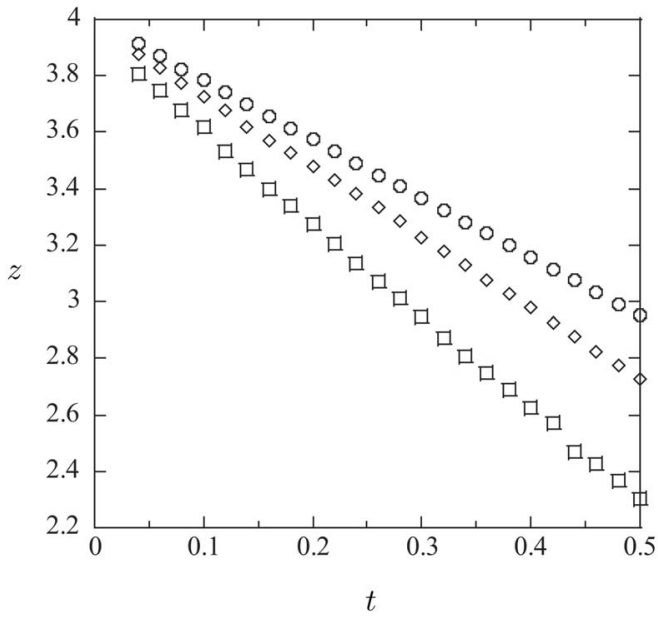

FIG. 9. Height of concentration quartiles, i.e., $c=\frac{1}{4}(\bigcirc), \frac{1}{2}(\diamond)$, and $\frac{3}{4}(\square)$, as functions of time for the same simulation as Fig. 8.

could not then be determined. Figure 9 plots the heights of the three quartiles as a function of time for a typical simulation. Each concentration quartile descends fairly linearly in time. One can see more clearly in this figure that by $t=0.5$ the front has descended at height 1.5 and has a thickness of around 1.0. Because the concentration profile in Fig. 8 varies faster near the $\frac{1}{4}$ concentration compared with the changes near the $\frac{3}{4}$ concentration, the thickness of the $\frac{1}{4}-\frac{1}{2}$ quartile in Fig. 9 is less than that of the $\frac{1}{2}-\frac{3}{4}$ quartile.

The thickness of the front $\delta$ is defined to be the difference in heights between the $\frac{1}{4}$ and $\frac{3}{4}$ concentrations. The growth of the thickness in time is plotted in Fig. 10 for the monodisperse simulation of Figs. 8 and 9. The apparent finite thickness of the front at the initial moment is smaller than the finest Fourier mode and probably due to this limited resolution. The best linear fit of the form $\delta \approx a t+b$ is plotted also in Fig. 10. Now, Mucha et al. ${ }^{12}$ have proposed a model in which the stratification of the front limits the size of eddies,

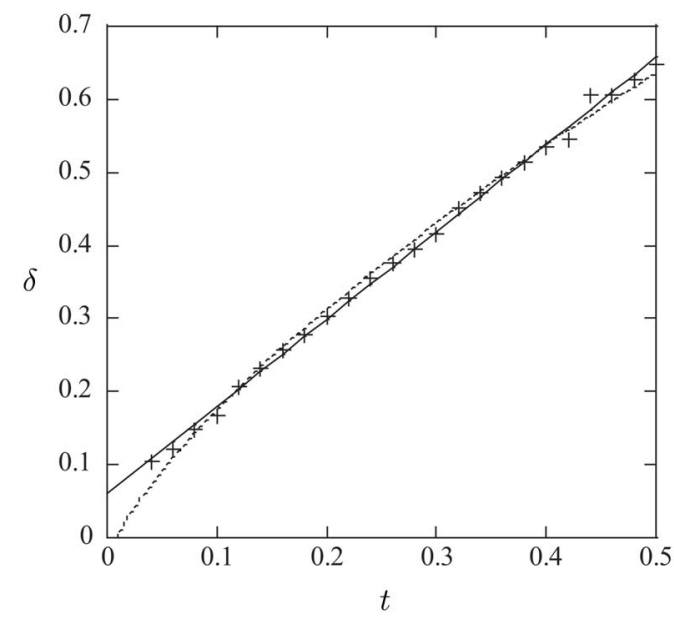

FIG. 10. Growth of thickness of the front in time for the same monodisperse simulation as Fig. 8. Also plotted are the best linear fit (solid line) and the best $t^{5 / 7}$ fit (dotted curve). 


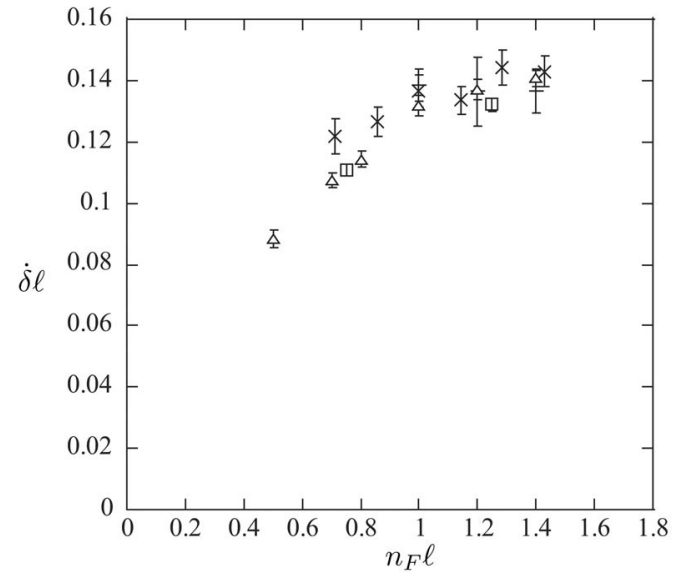

FIG. 11. Scaled rate of growth of the front $\dot{\delta} \ell$ as a function of the numerical resolution $n_{F} \ell$ for monodisperse simulations with sedimentation velocity $V_{0}=3$ and $N=500(+), 1372(\times), 4000(\triangle)$, and $6912(\square)$.

which yields a nonlinear diffusion equation with a diffusive growth of the front proportional to $t^{5 / 7}$. A best fit of the form $\delta \approx a t^{5 / 7}+b$ is also plotted in Fig. 10. The data from the numerical simulations seem to lack the curvature of the $t^{5 / 7}$ variation, agreeing better with the linear variation in time. For the rest of this paper, only the linear growth rate $\dot{\delta}$ will be considered. The values of the growth rate reported below will be the mean of eight independent runs, each run having a number of independent realizations. The different results between the runs allows error bars to be attached to the reported mean values.

In our earlier paper, ${ }^{13}$ the rate of growth of the front, i.e., $\dot{\delta}$, was found to scale inversely with the interparticle separation; i.e., $\ell=(4 / N)^{1 / 3}$. In that paper, we gave the following speculation on the origin of this scaling, using dimensional variables for the remainder of this paragraph. A cluster or blob of size $l$ would contain $n l^{3}$ particles with statistical fluctuations in the number of $\pm \sqrt{n l^{3}}$, where $n$ is the number density of the particles. Thus, there would be a fluctuation in the concentration of $\phi^{\prime}=\phi_{0} / \sqrt{n l^{3}}$. Now the extra weight of the blob $F \sqrt{n l^{3}}$ would in the Stokes regime produce an enhanced velocity $v^{\prime}=F \sqrt{n l^{3}} / 6 \pi \mu l \propto \sqrt{n l}$. Hence, the enhancement of the flux of particles from the concentration fluctuation $\phi^{\prime}$ falling at the extra velocity $v^{\prime}$ is $\overline{\phi^{\prime} v^{\prime}} \propto 1 / l$. The largest contribution to this flux enhancement comes from the smallest possible blobs; i.e., those with a size equal to the mean particle separation $n^{-1 / 3}$. The flux enhancement depletes the front, so leading to its spreading at a rate $\dot{\delta} \propto 1 / \ell \propto(N / 4)^{1 / 3}$, reverting to our nondimensional variables.

When the number of Fourier modes $4 n_{F}^{3}$ is set equal to the number of particles $N$, as in most of the simulations, the interparticle separation $\ell$ is precisely equal to $1 / n_{F}$. A number of monodisperse simulations have therefore been conducted in which the number of particles and number of Fourier modes were varied independently. Results for the growth rate multiplied by the interparticle separation $\dot{\delta} \ell$ are plotted in Fig. 11 as a function of the numerical resolution as given by the ratio of the interparticle separation divided by the smallest length resolved by the finest Fourier mode, as a function of $\ell n_{F}$. Once there are sufficient Fourier modes to resolve the interparticle separation, $1 / n_{F}<\ell$, the growth rate does not depend on the number of Fourier modes used. The growth rate is then given by

$$
\dot{\delta}=(0.141 \pm 0.003) / \ell,
$$

independent of the number of particles used in the simulation. In our earlier paper ${ }^{13}$ with fewer realizations to average over, we gave the growth rate as $0.12 / \ell$. As we noted previously, this scaling of the growth rate corresponds to heavierthan-average clusters falling faster, leaving the front depleted of particles, with the smallest clusters of size $\ell$ contributing most to this enhanced flux. The scaling $\delta=0.14 \ell^{-1} t$ is inconsistent with the nonlinear diffusion equation of Mucha et al. ${ }^{12}$ which has $\delta \propto \ell^{-3 / 7} t^{5 / 7}$.

Having demonstrated that our results are independent of the number of particles and number of Fourier modes used, we now consider the effects of polydispersity on the growth of the front. With the sedimentation velocity of the polydisperse particles being uniformly distributed between $V_{0}(1 \pm \alpha)$, the difference in velocities between the $\frac{3}{4}$ and $\frac{1}{4}$ of the population is $V_{0} \alpha$, which should be the rate of growth of the front as defined in this paper. On the other hand, the front in a monodisperse simulation growths at a rate of $0.14 / \ell$. Hence, in Fig. 12(a), the scaled rate of growth $\dot{\delta} \ell$ is plotted against the similarly scaled polydispersity $V_{0} \alpha \ell$. The results plotted in this form do not depend on the number of particles used in the simulations from $N=500$ to 10976 . In addition, different degrees of polydispersity, i.e., $2.5 \%$ and $5 \%$, with different sedimentation velocities from $V_{0}=0$ to 8.0 give the same results for equal $V_{0} \alpha \ell$. When the polydispersity is small, i.e., $V_{0} \alpha \ell<0.1$, it has little effect on the rate of growth of the front; i.e., $\dot{\delta} \approx 0.14 / \ell$. On the other hand, once the polydispersity would give just a little more growth of the front than the monodisperse case, i.e., $V_{0} \alpha \ell>0.2$, then the hydrodynamic interactions increase the growth little beyond the polydisperse growth alone; i.e., $\dot{\delta} \approx V_{0} \alpha$.

In Fig. 12(b), the rates of growth of the separate quartiles of the front are plotted as a function of the polydispersity. The thinner $\frac{1}{4}-\frac{1}{2}$ quartile grows entirely through the polydispersity once $V_{0} \alpha \ell>0.05$. On the other hand, the thicker $\frac{1}{2}-\frac{3}{4}$ quartile grows mainly through the effects of hydrodynamic interactions in the range of polydispersity studied.

Finally the concentration profiles of Fig. 8 are replotted in Fig. 13 in a frame moving with the mean sedimentation speed $V_{0}=3.0$ and rescaled by the growing thickness of the front: $\delta=1.388 t$. The profiles from the different times superpose, showing that the profiles adopt a similarity form. We have no theory for this form.

\section{CONCLUSION}

Both in experiments and in simulations, the thickness of the front was seen to grow linearly in time and not like $t^{5 / 7}$, as predicted by a proposed nonlinear diffusion equation. ${ }^{12}$ The growth rate was the same in three experimental cells with different cross sections. Similarly, the growth rate in the 

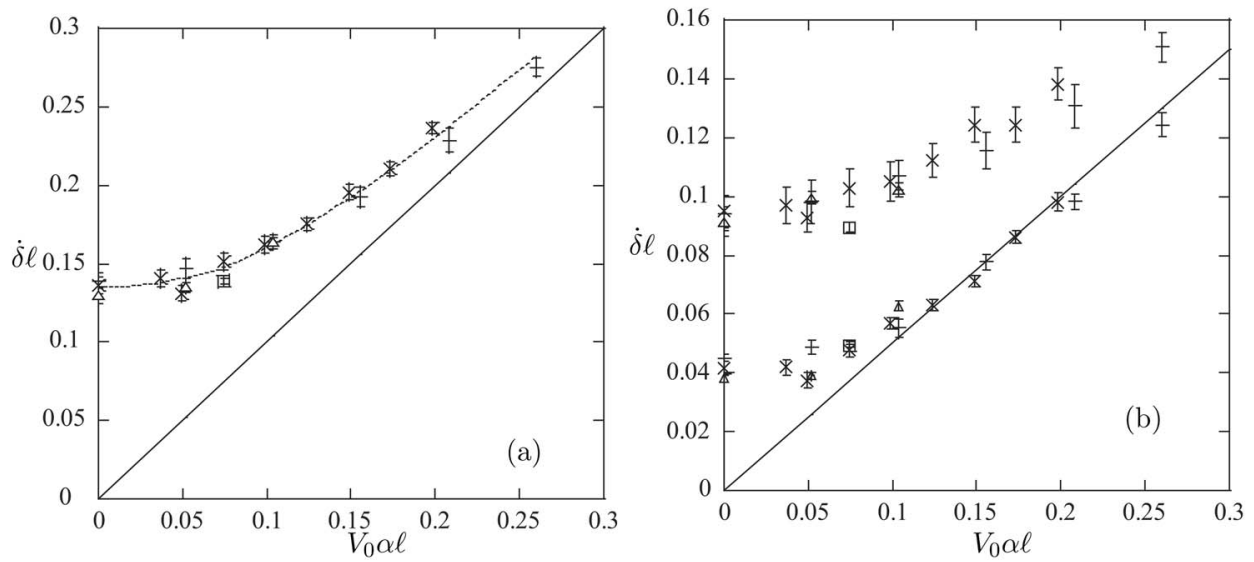

FIG. 12. (a) Scaled growth rate of the front $\dot{\delta} \ell$ as a function of the scaled polydispersity $V_{0} \alpha \ell$, with various $V_{0}$ and $\alpha$, and with $N=500(+), 1372(\times), 4000$ $(\triangle)$, and $10976(\square)$. The line $\dot{\delta}=V_{0} \alpha$ is the contribution of the polydispersity without hydrodynamic interactions. The dotted curve is explained in the conclusion. (b) Scaled rates of growth of the $\frac{1}{4}-\frac{1}{2}$ (lower data) and the $\frac{1}{2}-\frac{3}{4}$ (upper data) quartiles for the same simulations. The line $\dot{\delta}=V_{0} \alpha / 2$ is the contribution of the polydispersity without hydrodynamic interactions.

simulations was independent of the number of Fourier modes once they were sufficient to resolve the interparticle separation. This linear growth comes both from polydispersity in the size of the particles and from an enhanced flux with heavy blobs falling out of the front, a new effect revealed in the experiments and the simulations. In neither the experiments nor the simulations is there sufficient resolution to identify the hypothesized blobs. However, the front cannot become depleted unless heavier regions or blobs fall out of the front. These blobs may or may not have a long-time coherence.

For monodisperse suspensions, the simulations have rate of growth of the front in dimensionless form

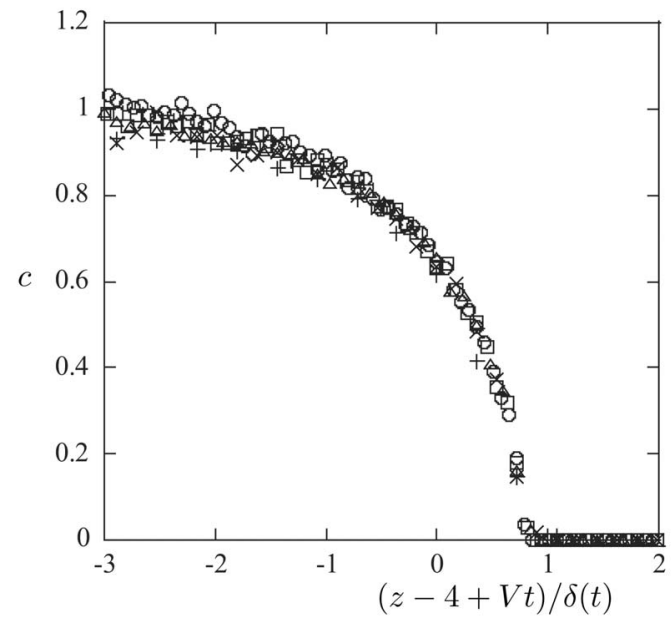

FIG. 13. Concentration profiles of Fig. 8 replotted in a frame moving with the mean sedimentation speed $V_{0}=3.0$ and rescaled by the growing thickness of the front $\delta=1.388 t$.

$$
\dot{\delta}=(0.141 \pm 0.003) / \ell
$$

which translates into the dimensional form

$$
\dot{\delta}=(1.65 \pm 0.04) V_{S} \phi^{1 / 3} \text {. }
$$

At a concentration of $\phi=0.3 \%$, this gives $0.24 V_{\mathrm{S}}$. The experiments, however, at $\phi=0.3 \%$ with the most monodisperse particles (batch B) found $0.14 V_{\mathrm{S}}$, a different value but similar magnitude. Moreover, the experiments with particle B did not see a $\phi^{1 / 3}$ dependence, but rather an increase to a maximum at an unexpectedly low $\phi=0.5 \%$, before a decrease due to an hindered settling. A similar maximum in the rate of spreading was found by Davis and Hassen, ${ }^{14}$ but at higher concentrations.

The effects on the spreading of the front from the polydispersity and from the enhanced flux were found not to be simply additive in both the experiments and simulations. Rather, the smaller of the two mechanisms had very little effect. Thus at $V_{0} \alpha \ell=0.08$, the polydispersity contributes only $7 \%$ to the final spreading, whereas it would have contributed $31 \%$ if the effects were simply additive. Similarly at $V_{0} \alpha \ell=0.20$ the enhanced flux contributes only $13 \%$, whereas it would have contributed $41 \%$ if the effects were simply additive.

In both the experiments and the simulations, the $\frac{1}{2}-\frac{3}{4}$ quartile was thicker than the $\frac{1}{4}-\frac{1}{2}$ quartile, up to twice as thick in the monodisperse case. Thus, the enhanced flux had a larger effect on the thicker $\frac{1}{2}-\frac{3}{4}$ quartile.

The nonlinear combination of the effects of polydispersity and hydrodynamics means that the simulations do not predict the simple $\phi^{1 / 3}$ concentration dependence, as suggested three paragraphs above. The results for the spreading of the front can be fitted by the ad hoc expression 


$$
(\dot{\delta})^{\beta}=k^{\beta}+\alpha^{\beta},
$$

with fitting parameters $k=0.135 \pm 0.002$ and $\beta=2.35 \pm 0.11$ (see dotted curve in Fig. 12). Translating this into dimensional terms gives

$$
\left(\dot{\delta} / V_{\mathrm{S}}\right)^{\beta}=\left(1.57 \phi^{1 / 3}\right)^{\beta}+\alpha^{\beta} .
$$

This fails to approximate the experimentally observed dependence on concentration for batch B particles. However, if the coefficient 1.57 is reduced to 1.0 and $\alpha$ is taken to be the measured 0.06, a reasonable prediction is obtained for the experimental results, as given by the dotted curve in Fig. 6.

Finally, the concentration profiles seem to adopt a universal form when viewed in a frame descending with the mean settling velocity and with distance scaled with the thickness of the front.

In summary, we have been able to quantify the effect of polydispersity and flux enhancement on the sedimentation front. Despite the gross simplification of the point-particle approach, we find a qualitative agreement between the simulations and the experiments.

\section{ACKNOWLEDGMENTS}

We wish to thank A. Daerr for his help in developing some IMAGEJ macros and F. Ratouchniak for technical assistance. Support from CONACyT is gratefully acknowledged by D.C.G.

${ }^{1}$ G. K. Batchelor, "Sedimentation in a dilute dispersion of spheres," J. Fluid Mech. 52, 245 (1972).

${ }^{2}$ R. E. Caflisch and J. H. C. Luke, "Variance in the sedimenting speed of a suspension,” Phys. Fluids 28, 759 (1985).
${ }^{3}$ E. J. Hinch, "Sedimentation of small particles," in Disorder and Mixing, edited by E. Guyon, J.-P. Nadal, and Y. Pomeau (Kluwer Academic, Dordrecht, 1988), p. 153.

${ }^{4} \mathrm{H}$. Nicolai and E. Guazzelli, "Effect of the vessel size on the hydrodynamic diffusion of sedimenting spheres," Phys. Fluids 7, 3 (1995).

${ }^{5}$ P. N. Segrè, E. Helbolzheimer, and P. M. Chaikin, "Long-range correlations in sedimentation," Phys. Rev. Lett. 79, 2574 (1997).

${ }^{6}$ G. Bernard-Michel, A. Monavon, D. Lhuillier, D. Abdo, and H. Simon, "Particle velocity fluctuations and correlation lengths in dilute sedimenting suspensions," Phys. Fluids 14, 2339 (2002).

${ }^{7}$ D. L. Koch and E. S. G. Shaqfeh, "Screening in sedimenting suspension," J. Fluid Mech. 224, 275 (1991).

${ }^{8}$ P. Tong and B. J. Ackerson, "Analogies between colloidal sedimentation and turbulent convection at high Prandtl numbers," Phys. Rev. E 58, R6931 (1998).

${ }^{9}$ J. H. C. Luke, "Decay of velocity fluctuations in a stably stratified suspension," Phys. Fluids 11, 754 (1999).

${ }^{10}$ S.-Y. Tee, P. J. Mucha, L. Cipelletti, S. Manley, M. P. Brenner, P. N. Segrè, and D. A. Weitz, "Nonuniversal velocity fluctuations of sedimenting particles," Phys. Rev. Lett. 89, 054501 (2002).

${ }^{11}$ P. J. Mucha, S.-Y. Tee, D. A. Weitz, B. I. Shraiman, and M. P. Brenner, "A model for velocity fluctuations in sedimentation," J. Fluid Mech. 501, 71 (2004).

${ }^{12}$ P. J. Mucha and M. P. Brenner, "Diffusivities and front propagation in sedimentation," Phys. Fluids 15, 1305 (2003).

${ }^{13}$ L. Bergougnoux, S. Ghicini, E. Guazzelli, and E. J. Hinch, "Spreading fronts and fluctuations in sedimentation," Phys. Fluids 15, 1875 (2003).

${ }^{14}$ R. H. Davis and M. A. Hassen, "Spreading of the interface at the top of a slightly polydisperse sedimenting suspension," J. Fluid Mech. 196, 107 (1988); R. H. Davis and M. A. Hassen, "Corrigendum," J. Fluid Mech. 202, 598 (1989)

${ }^{15}$ S. Lee, Y. Jang, C. Choi, and T. Lee, "Combined effect of sedimentation velocity fluctuation and self-sharpening on interface broadening," Phys. Fluids A 4, 2601 (1992).

${ }^{16}$ J. Martin, N. Rakotomalala, and D. Salin, "Hydrodynamic dispersion broadening of a sedimentation front," Phys. Fluids 6, 3215 (1994).

${ }^{17}$ As of January 2008, the software can be found at http://rsb.info.nih.gov/ij/. Author: Wayne Rasband (wayne@codon.nih.gov), Research Services Branch, National Institute of Mental Health, Bethesda, Maryland, USA. 